\title{
Ten years on and still no need to purchase a scanner
}

\author{
Dr Wayne Hirschowitz talks about his experience of using CT Dent \\ for the past ten years for dental scans for his implant practice.
}

\begin{abstract}
Why do you use a dental imaging centre? Practising in London and Kent, it is very convenient for patients to access a professional and quality imaging centre. This ensures consistency of images as well as having the additional facility of requesting a radiologist report. It is imperative for my implant practice to rely on the most modern imaging and trained staff so as to offer my patients the best possible care at reasonable prices. Accuracy, consistency and safety is of paramount importance.
\end{abstract}

\section{What made you choose CT Dent?}

I was introduced to CT Dent after researching the possibility of purchasing a scanner or using a bespoke facility. I did not believe the primary driver of any equipment acquisition is to recover capital costs. A patient will only be referred for a scan based on clinical need and not on economic grounds. In light of increasing litigation, in my experience having been in practice for over 30 years, a surgical dentist cannot have too much information when planning complex and often high risk surgery.

What kind of treatments do you refer patients for a CBCT scan for?

Primarily for my implant practice. However, when surgical removal of impacted, partially erupted or teeth necessitating removal and are close to strategic anatomical structures, a CT scan is mandatory to protect vital structures.

Have you had any feedback from your patients on the service?

In the ten years I've used CT Dent, I've been accustomed to patients complimenting the service they receive from the centre. There has never been an instance when a patient who arrives early or late is turned away. They are impressed by the efficiency of the service as well as the professionalism.
Are you happy with the service you have received from CT Dent over the past ten years? Absolutely yes.

\section{Do changes in technology make a difference} to you in terms of quality scans?

There have been significant advances in technology especially with regards to digital dentistry including digital scanners. I've changed my older software platform for a modern version with impressive improvement in quality.

\section{Do you or your patients have concerns over} radiation doses?

Absolutely. Therefore, it is important to convey accurate information to patients so they can make informed decisions regarding their treatment options. Explaining radiation doses and putting this into perspective especially when compared to medical scans, often allays fears, however, the radiation doses are not insignificant and this must be conveyed clearly to patients.

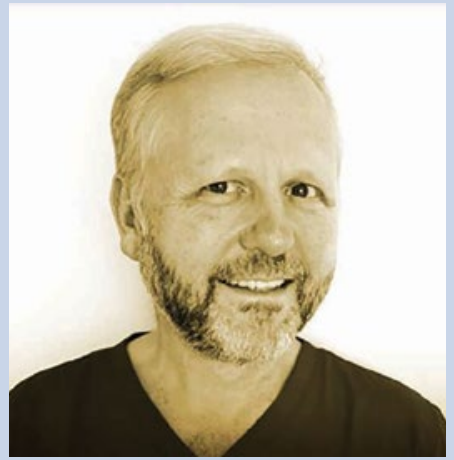

Dr Wayne Hirschowitz

Have you ever considered buying your own scanner?

As I'm reaching the latter part of my career, no. If I was embarking on an implant focused career, probably yes. I believe that purchasing new equipment should not be the primary economic driving force as this may lead to unnecessary use. However, as we all know, it has to be financially viable.

Dr Wayne Hirschowitz qualified in 1988 in Johannesburg, South Africa and went onto complete a postgraduate qualification in Forensic Odontology. After relocating to the UK in 1998, he entered into NHS/Private practice. He then went on to establish full private practices in Hertfordshire as well as Kent. He is the clinical director of Vantage Health supplying healthcare platforms to the NHS within medicine and dentistry. His clinical practice includes implantology as well as surgery and complex prosthodontic cases. In his spare time he performs forensic odontology cases for the police and coroner.

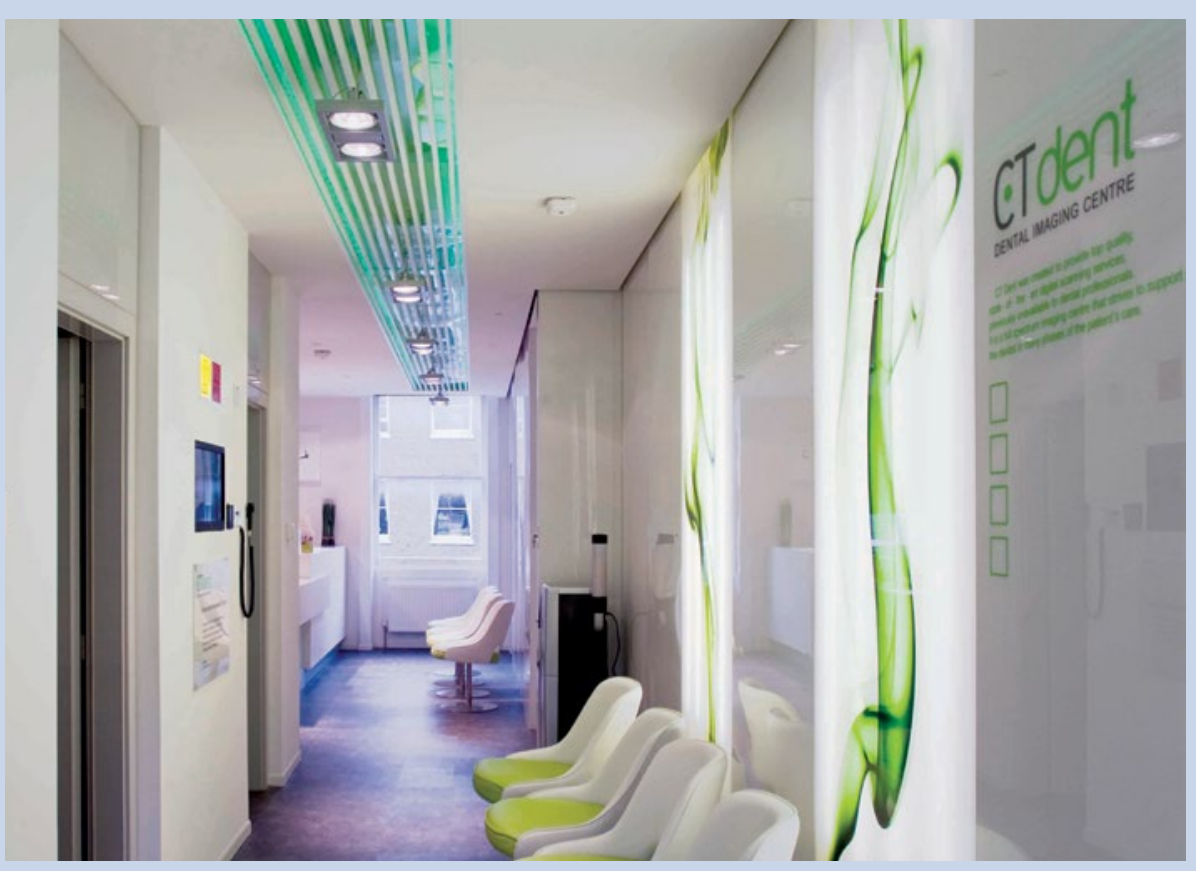

